Preprints of the

Max Planck Institute for

Research on Collective Goods

Bonn 2006/18

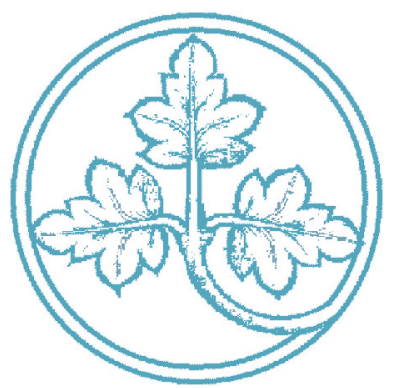

Informative Voting and the Samuelson Rule

Felix Bierbrauer / Marco Sahm

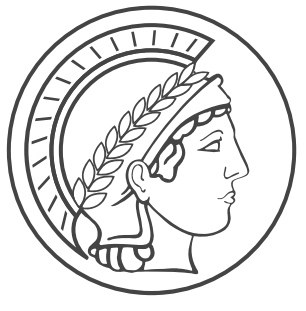




\title{
Informative Voting and the Samuelson Rule
}

\author{
Felix Bierbrauer / Marco Sahm
}

July 2006 


\title{
Informative Voting and the Samuelson Rule*
}

\author{
Felix Bierbrauer ${ }^{\dagger}$ \\ MPI Collective Goods, Bonn \\ Marco Sahm** \\ LMU München
}

This version: July 27, 2006

\begin{abstract}
We study the classical free-rider problem in public goods provision in a large economy with uncertainty about the average valuation of the public good. Individual preferences over public goods are shaped by a skill and a taste parameter. We use a mechanism design approach to solve for the optimal utilitarian provision rule. The relevant incentive constraints for information aggregation ensure that individuals behave as if they were engaging in informative voting over the level of public good provision. It is shown that the use of information by an optimal provision rule is inversely related to the polarization of preferences which results from the properties of the skill distribution.
\end{abstract}

Keywords: information aggregation, informative voting, public goods, two-dimensional heterogeneity

JEL: H41, D71, D72, D82

${ }^{*}$ We are very grateful for comments by Martin Hellwig, Klaus Schmidt, Thomas Gaube and Christoph Engel. We also thank participants of the Theory Workshop and the Public Economics Workshop at LMU Munich as well as of the SMYE 2005 at Geneva for useful suggestions.

${ }^{\dagger}$ Max Planck Institute for Research on Collective Goods, Kurt-Schumacher-Str. 10, 53113 Bonn, Germany. Email: bierbrauer@coll.mpg.de

** Lehrstuhl für Finanzwissenschaft, Ludwigstrasse 28 Vgb. III, 80539 München, Germany. Email: Marco.Sahm@lrz.uni-muenchen.de 


\section{Introduction}

We study a problem of optimal public good provision in a large economy in which individuals have private information on their valuation of the public good. Moreover, there is uncertainty about the average valuation of the of public good among individuals in the economy.

Private information on public goods preferences gives rise to the classical free-rider problem in public good provision. For economies with quasi-linear preferences, it is a well-known result that the tension between efficiency and incentive compatibility disappears as the number of individuals goes to infinity. This implies that, in an economy with infinitely many individuals, the first-best surplus-maximizing allocation can be implemented even if individuals have private information on their public goods preferences. ${ }^{1}$

In this paper, we question this result along the following line. First, with an infinite number of individuals, the underlying revelation game suffers from multiple equilibria. Second, selecting the truthful equilibrium, is implausible if confronted with considerations of tax incidence. Finally, we introduce a condition which is sufficient to avoid these outcomes. It is borrowed from the literature one voting mechanisms and interpreted as a requirement of informative voting. The main part of the paper is a characterization of the optimal rule for public good provision which satisfies this property.

\section{Mechanism Design in a large economy and tax incidence}

To illustrate the first point, recall that, in a finite economy with $N$ individuals, incentive compatibility requires that individual contributions to the cost of public good provision are commensurate to the individual's impact on the quantity decision. Otherwise individuals would not be willing to reveal their characteristics. In the limit case as $N \rightarrow \infty$, no single individual has a direct impact on public good provision. This implies that equal cost sharing is the only admissible scheme of public goods finance. As a consequence, there are multiple equilibria: all individuals pay equally for the public good and no single preference announcement has an effect on the chosen quantity, hence individuals are willing to make any conceivable announcement.

Given that the cost of public good provision has to be shared equally among individuals, we investigate how the well-being of individuals is affected by public good provision under this scheme of public goods finance (tax incidence). In particular, we use a simple model to show that the truthful equilibrium may have implausible consequences: individuals are assumed to

\footnotetext{
${ }^{1}$ It is a property of the Clarke-Groves mechanism, named after Clarke (1971) and Groves (1973), that a revelation of preferences is a dominant action for each individual and, moreover, that ex post budget balance is achieved almost surely as the number of individuals goes to infinity. If one uses the weaker solution concept of Bayes-Nash equilibrium then the first-best allocation is implementable even in a finite economy, d'Aspremont and Gérard-Varet (1979).
} 
affect the decision on public good provision in a way that runs counter to their own interests if public goods are financed via equal cost sharing.

To explain this point, a sketch of our model is helpful. Individuals possess private information on their effective valuation of the public good. The effective valuation is shaped by a taste parameter and a skill parameter. The taste parameter either takes a high or a low value. The skill parameter is drawn from a compact interval. It affects an individual's valuation of the public good because, under equal cost sharing, a less skilled individual suffers from a larger utility loss if forced to contribute to the cost of public good provision. Uncertainty about the distribution of public goods preferences equivalently, about the state of the economy - arises because the population share of individuals with a high taste parameter is a random variable. Under the requirement of equal cost sharing, the optimal utilitarian rule for public good provision is a modified version of the Samuelson rule which equates, in every state of the economy, the effective utilitarian valuation of the public good and the marginal cost of public good provision.

Suppose now that public goods are provided according to this rule and consider an individual with a high taste parameter but a very low skill level and, for the sake of concreteness, suppose this individual expects the state of the economy to be such that his own effective valuation of the public good is smaller than the effective utilitarian valuation. Consequently, this individual expects the level of the public good to be too large. If he chooses his taste announcement based on his preferred quantity decision he should announce a low taste realization. However, if all individuals with a low skill level refuse to reveal a high taste parameter, then the population share of individuals with a high taste parameter is biased downwards. But this implies that public goods cannot be provided according to the modified Samuelson rule, which requires the state of the economy to be known.

More generally, these considerations demonstrate that the Samuelson rule rests on the assumption that individuals reveal their taste parameter even though it would be in their interest to support a different outcome.

\section{The requirement of informative voting $(I V)$}

To deal with this issue we adopt the following approach: the revelation game is reinterpreted as a voting decision. More specifically, we interpret the announcement of a high (low) taste parameter as a vote in favor of a high (low) level of public good provision. Moreover, we assume that voting behavior is rational in the sense that an individual announces a high (low) taste parameter only if, under equal cost sharing, she prefers a rather high (low) level of the public good to be provided.

The assumption that individuals vote in favor of a high (small) provision level only if this is their preferred outcome translates into an additional constraint for the possibility to acquire information on the state of the econ- 
omy. The population share of individuals with a high taste parameter can be inferred from the distribution of votes only if the public good is indeed provided in such a way that all individuals with a high taste parameter prefer a large provision level over a small level and all individuals with a low taste parameter prefer a small level over a large level. We call these constraints the informative voting $(I V)$ constraints. $^{2}$

We characterize the optimal utilitarian provision rule which satisfies these $I V$ constraints. The main result is that the use of information by an optimal provision rule is inversely related to a specific measure for the polarization of effective valuations of the public good in the economy. ${ }^{3}$ To construct this measure, the economy is divided into two groups: the group of individuals with a high taste parameter and the group with a low taste parameter. Skill heterogeneity implies that there is within-group polarization of effective valuations for the public good; e.g. an individual with low skills and a high taste parameter has a small effective valuation relative to an individual with high skills and a high taste parameter.

As soon as there is some degree of within-group polarization, an optimal provision rule exhibits pooling; i.e. the same provision level is chosen for a whole range of possible states of the economy. In an extreme case of polarization, one finds that an optimal provision rule under $I V$ constraints is such that the same quantity of the public good has to be chosen in every state of the economy. In this sense, an optimal allocation makes no use of information.

\section{Sampling as a foundation for $I V$}

There exists no axiomatic foundation for the consideration of informative voting constraints. They are introduced into the analysis based on an analogy between voting schemes and the revelation game we started out with. Hence, they are different in status to the requirement of incentive compatibility, which is a necessary condition for the "implementability" of a scheme of public good provision if individuals have private information on their skill level and their taste parameter. By contrast, the $I V$ constraints are sufficient conditions which guarantee that the use of information on individual preferences is in line with the views of these individuals on the desirability of public good provision, given that the payment scheme prescribes equal cost sharing.

With respect to this concern, the $I V$ constraint are not unique; that is, one could imagine alternative sufficient conditions which serve the same pur-

\footnotetext{
${ }^{2}$ These constraints resemble the notion of informative voting which is used in the field of political economics, see e.g. Austen-Smith and Banks (1996).

${ }^{3}$ These observations are similar in spirit to classical results from the signalling literature. See e.g. Crawford and Sobel (1982), Schultz (1996), Grossman and Helpman (2001, Ch. 4).
} 
pose. The reason for focussing on this specific set of constraints is that they can be given a reasonable institutional interpretation: they determine the set of admissible rules of public good provision given that individuals vote over the level of public good provision and thereby take into account how the scheme of public goods finance affects the utility they derive from the public good.

To make this more precise, we finally study sampling mechanisms. A finite subset of $N$ randomly drawn individuals is asked to report their taste parameters. Based on these taste announcements, the mechanism designer estimates the effective utilitarian valuation of the public good and decides on public good provision. Finally, the cost of provision is shared equally among all individuals in the economy. We investigate the properties of an optimal mechanism as the sample size $N$ grows and individual influence on the level of public good provision disappears. We show that as $N \rightarrow \infty$ the optimal provision rule under sampling converges to the optimal provision rule in the original mechanism design problem under $I V$ constraints. $^{4}$

The crucial assumption for our study of provision rules based on sampling is that the payment scheme treats sampled individuals not differently than individuals who possess the same characteristics but have not been in the sample. From a general mechanism design perspective, this clearly involves a loss of generality. There certainly exist welfare superior allocation mechanisms that do not share this property but force sampled individuals to internalize the consequences of their announcements for the whole economy. ${ }^{5}$

Our convergence result establishes that the role of the $I V$ constraints really is to shift the focus to considerations of tax incidence. We treat equal cost sharing as given. In particular, this implies that an individual's contribution to the cost of public good provision does not depend on the probability of being pivotal for the decision on public good provision. Consequently, only the scheme of taxation shapes individual preferences on public good provision. Given these preferences, we then need to structure incentives such that information on the distribution of taste parameters becomes available for the decision on public good provision.

\footnotetext{
${ }^{4}$ As a corollary of this analysis, we show that the optimal provision rule under $I V$ constraints provides an upper bound to the welfare levels which are achievable under sampling, for any finite sample size $N$. Put differently, we show that an optimal sample size does not exist. This observation is interpreted as a version of the famous Condorcet Jury Theorem. A discussion of this theorem and of related results can be found in Piketty (1999).

${ }^{5}$ As a consequence our approach differs from the sampling mechanisms analyzed by Green and Laffont (1979, Ch.12) and Gary-Bobo and Jaaidane (2000). While these authors study also allocation problems using only a subset of individuals for information aggregation, they assume that contributions to the cost of public good provision may differ for individuals within the sample and those who are not in the sample.
} 


\section{Relationship to the theory of optimal income taxation}

The initial motivation of this paper was to study more generally, optimal rules for income taxation and public good provision in an economy where individuals have differing levels of ability and differing tastes for public goods. ${ }^{6}$ Even though this paper focusses on the conceptual issues that arise in a large economy with private information on public goods preferences, it still provides a link between these two branches of the literature. It characterizes the optimal rule for public good provision in the following environment: Individuals derive utility from a public good, a private consumption good and leisure. Moreover, the utility function is additively separable and quasilinear in leisure. ${ }^{7}$ The final allocation is determined sequentially. First, the level of public good provision is determined. This generates a revenue requirement in the public sector budget constraint. Second, the income tax schedule is chosen optimally, subject to this predetermined revenue requirement.

The optimal provision for public goods rule derived in section 3 of this paper is also optimal in this extended model under the assumption that, once the level of public good provision is fixed, tax authorities choose an optimal non-linear income tax in order to finance these expenditures. ${ }^{8}$

The remainder of this paper is organized as follows. In Section 2 we derive the mechanism design problem under $I V$ constraints. In Section 3 the solution to this problem is characterized. Section 4 contains the discussion of sampling mechanisms and the derivation of the Condorcet Jury Theorem. The last section contains concluding remarks. All proofs can be found in the appendix.

\footnotetext{
${ }^{6}$ Heterogeneity with respect to earning abilities is underlying the equity-efficiency tradeoff studied in the theory of optimal income taxation in the tradition of Mirrlees (1971). Heterogeneity with respect to public goods preferences drives the literature on the freerider problem in public goods provision.

${ }^{7}$ An optimal income tax in this setting has been characterized by Weymark (1986, 1987).

${ }^{8}$ In particular, tax authorities do not distort the optimal income tax in order to mitigate the welfare burden of the incentive constraints that are relevant for the decision on public good provision; that is, tax authorities cannot commit not to use an optimal income tax once the revenue requirement has been determined. However, as shown in Bierbrauer (2005b,c), if such a commitment was possible, it would, in general, lead to welfare superior outcomes.
} 


\section{The Model}

\subsection{The Environment}

The economy consists of a continuum of individuals $i \in I:=[0,1]$. Individuals differ with respect to their skill level $w^{i}$, and the utility they derive from a public good. The latter depends on a taste parameter $\theta^{i}$ that may take two different values:

$$
\theta^{i} \in \Theta:=\left\{\theta_{L}, \theta_{H}\right\} \quad \text { with } \quad 0 \leq \theta_{L}<\theta_{H} .
$$

The skill parameter belongs to the compact interval

$$
w^{i} \in W:=[\underline{w}, \bar{w}] \quad \text { with } \quad 0<\underline{w} \leq \bar{w} .
$$

Agent $i$ 's utility function is given by

$$
U^{i}=\theta^{i} Q-\frac{t^{i}}{w^{i}}
$$

$Q$ denotes the quantity of a non-excludable public good and $t^{i}$ captures $i$ 's contribution to the cost of public good provision. Note that a lower skill level implies a larger utility loss from a given payment obligation. The underlying idea is that, for less able individuals, it is harder to generate the income needed to meet a given payment obligation.

The function $U^{i}$ is the cardinal representation of preferences which is relevant for welfare assessments. An individual's ranking of alternatives can be equivalently expressed by the monotone transformation

$$
w^{i} U^{i}=\theta^{i} w^{i} Q-t^{i} .
$$

We refer to the product $\theta^{i} w^{i}$ as individual $i$ 's effective valuation of the public good.

The parameters $w^{i}$ and $\theta^{i}$ are both private information of individual $i$ and taken to be the realizations of the stochastically independent random variables $\tilde{w}^{i}$ and $\tilde{\theta}^{i}$, respectively. The random variables $\left\{\tilde{w}^{i}\right\}_{i \in I}$ are independently and identically distributed (i.i.d.). Their probability distribution is represented by a cumulative distribution function $F: W \rightarrow[0,1]$ with density $f$. The random variables $\left\{\tilde{\theta}^{i}\right\}_{i \in I}$ are also i.i.d.. By $p$ we denote the probability that any one individual has a high taste parameter,

$$
p:=\operatorname{Prob}\left\{\theta^{i}=\theta_{H}\right\} .
$$

In addition, we assume that a law of large numbers (LLN) applies; ${ }^{9}$ that is, almost surely, after the realization of randomness at the individual level,

\footnotetext{
${ }^{9}$ Postulating a LLN for a continuum of i.i.d. random variables creates a measurability problem, as has been noted by Judd (1985) and Feldman and Gilles (1985). There is, however, a recent literature on modeling approaches which circumvent this measurability problem; see Alòs-Ferrer (2002) or Al-Najjar (2004).
} 
the cross-section distribution of characteristics in the economy coincides with the ex ante probability distribution that governs the randomness at the individual level. Accordingly, the value $F(w)$ and the probability $p$ are interpreted as the fractions of individuals with earning ability $w^{i} \leq w$ and a high taste parameter, respectively. The $L L N$ also implies that the empirical skill distribution and the empirical taste distribution are independent; that is, on every subinterval $\left[w^{\prime}, w^{\prime \prime}\right] \subset W$ of the support of the skill distribution, the share of individuals with a high taste parameter is equal to $p$.

We assume that the distribution $F$ is common knowledge. Consequently, at the aggregate level, there is no uncertainty about the skill distribution. By contrast, the share of individuals with a high taste parameter $p$ is taken to be a random quantity; i.e. there is uncertainty with respect to the average valuation of the public good.

To sum up, the information structure has a known skill distribution and aggregate uncertainty with respect to the taste parameters. The unknown parameter $p$ is henceforth also referred to as the state of the economy. It is the relevant object for the process of information aggregation.

\section{Incentive Compatible Allocation Rules in a Continuum Economy}

We limit attention to anonymous and incentive compatible allocation rules. An anonymous allocation rule $(Q, t)$ consists of a provision rule for the public good and a payment scheme to cover the cost of provision.

The provision rule $Q$ assigns to alternative values of $p$ a quantity of the public good,

$$
Q:[0,1] \rightarrow \mathbb{R}_{+}, \quad p \mapsto Q(p) .
$$

This provision rule is anonymous in the sense that the level of provision $Q(p)$ depends only on the distribution of characteristics in the economy. It does not depend on the skill and taste parameters of specific individuals. ${ }^{10}$ The payment scheme $t$ specifies for each individual $i$ a payment obligation as a function of the distribution of characteristics in the economy $p$ and individual $i$ 's characteristics $\left(\theta^{i}, w^{i}\right)$. The payment scheme is anonymous in the sense that individuals with the same characteristics have the same payment obligation in every state $p$ of the economy. Put differently, individual payments do not depend on the index $i$. Formally the payment scheme is described as a function

$$
t:[0,1] \times \Theta \times W \rightarrow \mathbb{R},(p, \theta, w) \mapsto t(p, \theta, w) .
$$

Individuals have private information on their skill and their taste parameter. This gives rise to the following incentive compatibility constraints.

\footnotetext{
${ }^{10}$ Guesnerie (1995) calls this property anonymity in influence.
} 
Definition 1 An anonymous allocation rule is called incentive compatible $(I C)$ if $\forall p \in[0,1], \forall(\theta, w) \in \Theta \times W$, and $\forall(\hat{\theta}, \hat{w}) \in \Theta \times W$,

$$
\theta w Q(p)-t(p, \theta, w) \geq \theta w Q(p)-t(p, \hat{\theta}, \hat{w}) .
$$

These incentive constraints are to be read as follows: Suppose that a mechanism designer wants to implement an allocation rule $(Q, t)$. In a revelation game, he collects data from all individuals on their skill and on their taste parameter. The collection of these announcements is then used for two purposes: first, the profile of all taste announcements $\left(\hat{\theta}^{i}\right)_{i \in I}$ is used to deduce the actual value of $p$; second, for given $p$, the individual announcement $\left(\hat{\theta}^{i}, \hat{w}^{i}\right)$ is used to determine the payment obligation of individual $i$. The requirement of incentive compatibility deals with this second step only. It ensures that, for a given state $p$, an individual is indeed willing to make the payment prescribed by the payment scheme $t$. Put differently, an individual has no reason to hide her characteristics in order to achieve a preferred treatment by the payment scheme.

These $I C$ constraints have to be satisfied for each possible value of $p$. Put differently, whatever the "announced state of the world" which arises under the first step, any individual is willing to reveal her characteristics; i.e we consider implementation in dominant strategies.

As we consider a continuum economy, no single individual has a direct impact on the "announced state of the world". This is reflected in the fact that the same level of $p$ appears on the left hand side and the right hand side of the $I C$ constraint. As a consequence, no single individual has a direct impact on the level of public good provision. Individuals are concerned only with a minimization of their payment obligation. This gives rise to the classical free-rider problem. As access to the public good is free, no one is willing to pay more than he is forced to. ${ }^{11}$ These observations yield the following characterization of incentive compatible allocation rules.

Lemma 1 The following statements are equivalent.

1. $(Q, t)$ is $I C$.

2. $(Q, t)$ satisfies $\forall p, \forall(\theta, w)$ and $\forall(\hat{\theta}, \hat{w}), t(p, \theta, w)=t(p, \hat{\theta}, \hat{w})$.

Consequently, any $I C$ payment scheme is constant in the sense that, for given $p$, all individuals are treated equally. The converse statement is also

\footnotetext{
${ }^{11}$ Note that if a balanced budget has to be achieved and there are limits to coercion due to participation constraints as in Mailath and Postlewaite (1990) or in Hellwig (2003), as well as individuals who do not value the public good at all - i.e. with effective valuation of 0 - one will end up with $Q \equiv 0$ under any admissible, incentive compatible allocation rule.
} 
true; that is, any anonymous provision rule $Q: p \mapsto Q(p)$ gives rise to an $I C$ allocation rule if accompanied by constant payments, i.e. a payment scheme that does only depend on $p$.

\section{Budget Balance and Incentive Compatibility}

The per capita cost of public good provision is given by a twice continuously

differentiable, strictly increasing and strictly convex cost function $K: \mathbb{R}_{+} \rightarrow$ $\mathbb{R}_{+}$, which satisfies $K(0)=0$ as well as the boundary conditions

$$
\lim _{x \rightarrow 0} K^{\prime}(x)=0 \text { and } \lim _{x \rightarrow \infty} K^{\prime}(x)=\infty .
$$

The payment scheme has to be such that the costs of public good provision are covered, i.e. such that aggregate payments are equal to the cost of provision. Combining the requirement of a balanced budget with the requirement of $I C$ yields the following observation.

Lemma 2 An anonymous allocation rule $(Q, t)$ satisfies $I C$ and a balanced budget if and only if the payment scheme is such that

$$
\forall p \in[0,1], \forall(\theta, w) \in \Theta \times W: \quad t(p, \theta, w)=K(Q(p)) .
$$

Lemma 2 allows us to represent an individual's assessment of an allocation rule $(Q, t)$, which is budgetary feasible and incentive compatible, in the following reduced form, which depends only on the provision rule $Q$,

$$
U\left(p, \theta^{i}, w^{i}\right):=\theta^{i} Q(p)-\frac{K(Q(p))}{w^{i}} .
$$

Remark Using results from Weymark (1986, 1987), this reduced form can also be derived from the following extended model:

i) The informational structure is as described above. Individual preferences, however, are defined over a public good $Q$, private goods consumption $C$, and foregone leisure $L=Y / w^{i}$, where $Y$ denotes effective labor supply or income. Preferences of individual $i$ can be represented by the following utility function

$$
\theta^{i} Q+u(C)-\frac{Y}{w^{i}}
$$

where $u$ is an increasing and strictly concave function.

ii) The decisions on public good provision and taxation are made sequentially. First, the level of public good provision is determined. Then, given $Q$, an income tax schedule is chosen in order to maximize utilitarian welfare subject to incentive compatibility constraints and a tax revenue requirement of $K(Q)$. 


\section{Expected utilitarian welfare}

In what follows, we consider mechanism design problems of a benevolent utilitarian planner. A budgetary-feasible and incentive-compatible allocation is evaluated from an ex ante perspective, i.e. before the actual value of $p$ is known. For simplicity, we impose the following assumption on the prior of the mechanism designer. ${ }^{12}$

Assumption 1 The mechanism designer takes $p$ to be the realization of a random variable which is uniformly distributed on $[0,1]$.

Under Assumption 1 and the $L L N$, ex ante expected utilitarian welfare becomes

$$
\begin{aligned}
E W & :=\int_{0}^{1}\left\{\left[p \theta_{H}+(1-p) \theta_{L}\right] Q(p)-\left[\int_{\underline{w}}^{\bar{w}} \frac{f(w)}{w} d w\right] K(Q(p))\right\} d p \\
& =\lambda \int_{0}^{1}\{\bar{v}(p) Q(p)-K(Q(p))\} d p
\end{aligned}
$$

where $\lambda:=\int(1 / w) f(w) d w$ is an index of the marginal welfare effects of the cost of public good provision under equal cost sharing and

$$
\bar{v}(p):=\frac{p \theta_{H}+(1-p) \theta_{L}}{\lambda}
$$

is the effective utilitarian valuation of the public good.

\subsection{Information Aggregation}

The problem of information aggregation is concerned with the question whether a mechanism designer is able to learn how many individuals in the economy have a high taste parameter. Recall that the mechanism designer evaluates the profile of taste announcements $\left(\hat{\theta}^{i}\right)_{i \in I}$ to learn the actual value of $p$. Hence, he gets to know the actual state of the world if and only if (almost) all individuals reveal their taste parameter truthfully.

However, under an incentive-compatible and budgetary-feasible allocation rule individuals are indifferent which taste parameter to announce; i.e. the revelation game suffers from a problem of multiple equilibria. The reason is that we consider only anonymous allocation mechanisms. Consequently, no individual has a direct impact on public good provision. In addition, incentive compatibility requires that the payment scheme treats all individuals alike. These two facts imply that individuals are willing to make any announcement in the underlying revelation game.

\footnotetext{
${ }^{12}$ Throughout we do not need to impose a common prior assumption. We only specify the prior beliefs of the mechanism designer.
} 
In particular, this implies that individuals are willing to announce any taste parameter. The problem of information aggregation, however, is resolved only if all individuals announce their taste parameter truthfully. We will now argue that such an obedient behavior cannot be taken for granted.

\section{A problem with the Samuelson Rule}

To illustrate this point, we discuss the provision rule $Q^{*}: p \mapsto Q^{*}(p)$, which is chosen by a utilitarian planner who maximizes $E W$ pointwise; i.e. who maximizes the expression $\bar{v}(p) Q(p)-K(Q(p))$ for every $p \in[0,1]$. This provision rule $Q^{*}$ is nothing but the Samuelson rule under equal cost sharing. It is characterized by a continuum of first order conditions

$$
\forall p: \quad \bar{v}(p)=K^{\prime}\left(Q^{*}(p)\right) .
$$

For brevity, we also refer to $Q^{*}$ as the first best provision rule. Under $Q^{*}$ individual preferences about the "announced state of the world" result from the reduced form

$$
U^{*}(p, \theta, w):=\theta Q^{*}(p)-\frac{K\left(Q^{*}(p)\right)}{w} .
$$

It is easily verified that

$$
U_{p}^{*}(p, \theta, w)=\frac{1}{w} Q^{* \prime}(p)(\theta w-\bar{v}(p))\left\{\begin{array}{lll}
<0 & \text { if } & \theta w<\bar{v}(p), \\
=0 & \text { if } & \theta w=\bar{v}(p), \\
>0 & \text { if } \quad \theta w>\bar{v}(p) .
\end{array}\right.
$$

Under provision rule $Q^{*}$ an individual prefers a larger level of $p$ - or equivalently a larger level of public good provision - if and only if his effective valuation exceeds the effective utilitarian valuation. Likewise an individual with a below average effective valuation below the average prefers to have a lower quantity of the public good.

These observations imply that an individual would refuse to reveal his taste parameter if he believed he had an influence on the decision on public good provision. To see this, consider an individual with a low taste parameter and a high skill level who has an effective valuation close to $\theta_{L} \bar{w}$. Moreover, for the sake of concreteness, assume that this individual believes $p$ to be very low. ${ }^{13}$ If a vast majority of individuals have a low taste parameter, then this individual can be sure that his own effective valuation lies above the average, $\theta_{L} \bar{w}>\bar{v}(p)$. Put differently, under $Q^{*}$, the individual in question expects that the quantity of the public good is too low. As a consequence, the individual would be happy if the mechanism designer perceived $p$ to be larger. Hence, this individual is inclined to announce a high taste parameter in order to "contribute" to a more preferred outcome.

\footnotetext{
${ }^{13}$ When this individual decides ex interim what taste parameter to announce, her prior beliefs put a lot of probability mass on values of $p$ which are close to zero.
} 


\section{The Informative Voting Constraints}

The point of these considerations is that, even though individuals have no direct influence on public good provision, they are not indifferent about the mechanism designer's perception of $p$. That is, they are not indifferent regarding the outcome of the revelation game.

We now state a formal condition, called informative voting $(I V)$, that we impose on the mechanism design problem. It is inspired by game-theoretic models of voting decisions in the field of political economy. For the moment, we just introduce these conditions and provide an interpretation. However, in Section 4 we discuss a more rigorous theoretical foundation.

In our setting $I V$ ensures that individuals are "really" willing to reveal their taste parameter; that is, the $I V$ constraints guarantee that individuals are not tempted to break the indifference among all conceivable taste announcements such that they "contribute" to a more preferred perception of the state of the economy by falsely expressing their own preferences.

Definition 2 A provision rule $Q$ is said to satisfy the $I V$ property if the following holds for any $w \in W$ and any $p \in[0,1]: U\left(p, \theta_{L}, w\right)$ is nonincreasing in $p$ and $U\left(p, \theta_{H}, w\right)$ is non-decreasing in $p$.

These monotonicity constraints are referred to as $I V$ constraints because of the idea that any individual subscribes to one of two groups, either to those individuals with $\theta^{i}=\theta_{L}$ or to those with $\theta^{i}=\theta_{H}$. Informative voting hence is a sufficient condition which ensures that each individual supports the group which shares her own taste parameter. As a consequence of this behavior, the distribution of votes makes it possible to deduce the actual value of $p$.

We interpret the $I V$ constraints as a condition of robustness ${ }^{14}$ that is, they ensure that, whatever the prior beliefs of individuals on the likelihood of different values of $p$, no individual has a reason to report a false taste parameter in order to "contribute" to a more favorable perception of $p$.

\section{Mechanism Design under $I V$ constraints}

We can now define the mechanism design problem of a utilitarian planner who has to choose an $I C$ allocation rule $(Q, t)$ and, in addition, uses the $I V$ constraints to ensure that he can deduce the actual value of $p$ from the profile of taste announcements $\left(\hat{\theta}^{i}\right)_{i \in I}$ in the revelation game.

\footnotetext{
${ }^{14}$ Further discussion of this notion can be found in Bergemann and Morris (2005), Chung and Ely (2004) or Kalai (2004). For mechanism design problems with private values, the notions robustness and implementation in dominant strategies are equivalent.
} 
Definition 3 The following problem is called the informative voting problem $(P)$ : Choose a provision rule $Q$ in order to maximize $E W$ subject to the $I V$ constraints. The solution to this problem is denoted by $Q^{* *}$, the induced optimal welfare level by $E W^{* *}$.

In Section 4 we provide a theoretical foundation for this mechanism design problem under $I V$ constraints. There, we show that the optimal provision rule $Q^{* *}$ can be interpreted as the limit outcome of a sequence of mechanism design problems with vanishing individual influence on public good provision. Before turning to this issue, we characterize the solution of problem $P$.

\section{Optimal Provision under Informative Voting}

In this section we characterize the solution to the informative voting problem. The key insight is that the extent to which the optimal provision rule $Q^{* *}$ reflects variations in the average valuation of the public good $p$ depends on a specific measure of preference polarization. The role of skill heterogeneity for preference polarization is easily demonstrated with the following alternative characterization of the $I V$ property.

Lemma 3 A provision rule $Q$ satisfies $I V$, if and only if the following two properties hold for any pair $p, p^{\prime} \in[0,1]$ with $p^{\prime}>p$ :

i) Q is increasing: $Q\left(p^{\prime}\right) \geq Q(p)$.

ii) If $Q\left(p^{\prime}\right)>Q(p)$, then

$$
\theta_{H} \underline{w} \geq \frac{K\left(Q\left(p^{\prime}\right)\right)-K(Q(p))}{Q\left(p^{\prime}\right)-Q(p)} \geq \theta_{L} \bar{w} .
$$

A proof of the Lemma can be found in section A.3 of the Appendix. It establishes that, with a monotonically increasing provision rule, informative voting is ensured if and only if the following two conditions are met: an individual with the top skill level and a low taste parameter wants the level of public good provision to be as small as possible and an individual with the minimal skill level but a high taste parameter wants it to be as large as possible. Intuitively, among all individuals with a low taste parameter, the effective valuation of the public good is highest for the individual with the highest skill level. If even this individual wants the public good to be small, then the same is true for every individual with a low taste parameter. A similar reasoning applies to the set of individuals with a high taste parameter. These observations allow to prove the following proposition; see section A.3. 


\section{Proposition 1}

i) If $\theta_{L} \bar{w}>\theta_{H} \underline{w}$, then a provision rule $Q$ satisfies the $I V$ property if and only if it is constant: for all $p, Q(p)=\bar{Q}$, for some $\bar{Q} \in \mathbb{R}_{+}$.

ii) The first best provision rule $Q^{*}$ satisfies the $I V$ constraints if and only if there is no skill heterogeneity, i.e. $\underline{w}=\bar{w}$.

Part i) of Proposition 1 follows immediately from Lemma 3. It establishes that the requirement of $I V$ may indeed heavily restrict the set of admissible allocation rules. If $\theta_{L} \bar{w}>\theta_{H} \underline{w}$, then a provision rule satisfies $I V$ if and only if information aggregation plays no role.

We refer to a parameter constellation with $\theta_{L} \bar{w}>\theta_{H} \underline{w}$ as a situation of large within-group polarization. This terminology reflects the following considerations: The $I V$ constraints essentially require that all individuals with the same taste parameter have the same views on public good provision. However, individuals who have the same taste parameter differ in their effective valuations of the public good due to skill heterogeneity. We take the distance $\bar{w}-\underline{w}$ to be a measure of within-group polarization. It is said to be large if the group of individuals with low taste parameter contains individuals whose skill level is so high that their effective valuation exceeds the one of low skilled individuals in the high taste group.

A parameter constellation with $\underline{w}=\bar{w}$ is one in which there is no withingroup polarization at all. This implies that the $I V$ constraints do not have any bite and that the first best provision rule $Q^{*}$ is admissible. To be clear, there are conflicting interests between groups but these conflicts are no impediment for information aggregation. For any $p$, low taste individuals have an effective valuation for the public good which falls short of the average effective valuation. Likewise high taste individuals have an effective valuation which exceeds the average level. Hence, if one decides on public good provision according to the average effective valuation, one ends up in a situation where the provision level is too low for the high taste group and too high for the low taste group. But this is exactly the pattern of views on public good provision which is postulated by the $I V$ constraints.

The results in Proposition 1 characterize the optimal provision rule under $I V$ constraints for the extreme cases of no within-group polarization and large within-group polarization. For the remainder of this section we consider parameter constellations of moderate within-group polarization which satisfy $\underline{w} \neq \bar{w}$ and $\theta_{L} \bar{w} \leq \theta_{H} \underline{w}$. The key observation is that the second best provision rule $Q^{* *}$ is characterized by pooling; that is, $Q^{* *}$ is not strictly increasing over the whole domain $[0,1]$.

Proposition 2 Let $\underline{w}<\bar{w}$. A provision rule which solves the informative voting problem $P$ is either constant or has two, three or four pooling levels. 
The proof of Proposition 2 can be found in section A.1 of the appendix. The appendix also contains a formal definition of the terminology provision rule with two/three/four pooling levels. Here, we illustrate these provision rules graphically; see figures 1 and 2 below.

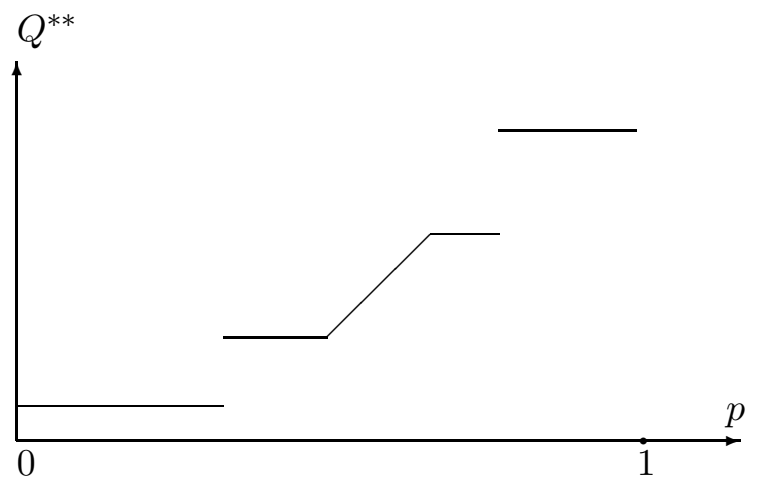

Figure 1: The figure depicts a provision rule with four pooling levels. Over an intermediate range $Q^{* *}$ is strictly increasing and coincides with the first-best provision rule $Q^{*}$. The graph assumes a quadratic cost function which implies that $Q^{*}$ is a linear function of $p$.

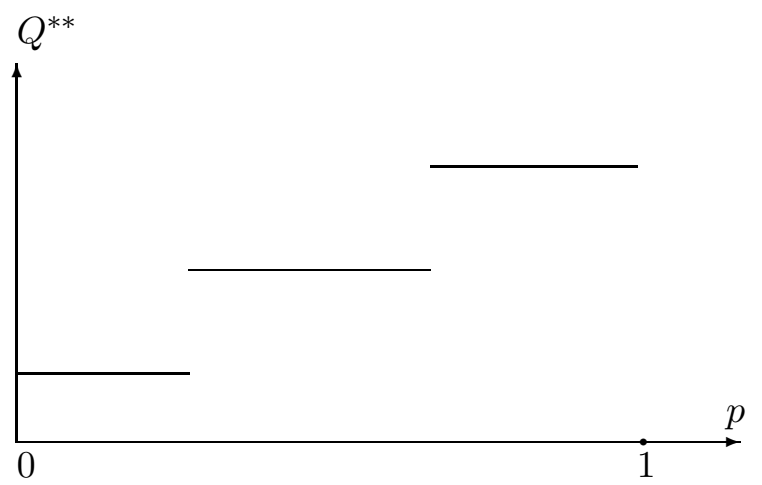

Figure 2: The figure depicts a provision rule with three pooling levels. A provision rule with two pooling levels looks similar, except that the image of $Q^{* *}$ consists only of two elements.

The proof of Proposition 2 is based on the observation that, under $I V$ constraints, the image of a provision rule contains at most one element smaller than the most preferred provision level of an individual with effective valuation $\theta_{L} \bar{w}$, which we denote by $\bar{Q}_{L}$, and at most one provision level larger than the most preferred provision level of an individual with effective val- 
uation $\theta_{H} \underline{w}$, denoted by $Q_{H} \cdot{ }^{15}$ Moreover, under moderate within-group polarization, $Q^{*}(0)$ is smaller than $\bar{Q}_{L}$ and $Q^{*}(1)$ is larger than $\bar{Q}_{L}$. This allows us to show that an optimal provision rule under $I V$ constraints has exactly one element smaller than $\bar{Q}_{L}$ and exactly one element larger than $\underline{Q}_{H}$. These considerations single out provision rules with four, three and two pooling levels as candidate solutions.

Intuitively, in the light of Propositions 1 and 2, one expects that the second best provision rule $Q^{* *}$ looks similar to the first best provision rule $Q^{*}$ if within-group polarization is relatively mild and becomes more similar to a constant provision rule as within-group polarization increases. Put differently, if polarization is mild one expects the optimal provision rule to have four pooling levels and a large interval over which $Q^{* *}$ coincides with $Q^{*}$. If within-group polarization becomes more pronounced one expects the optimal provision rule to have two pooling levels.

We refrain from providing a general proof for these intuitive statements. This would require an awkward exercise, distinguishing a variety of assumptions on the parameters $\theta_{L}, \theta_{H}, \bar{w}, \underline{w}$ and $\lambda$, i.e. the skill distribution $F$. We only provide an example that allows us to verify this intuition.

Example Suppose $K(Q)=\frac{1}{2} Q^{2}, \theta_{L}=1, \theta_{H}=3$, and $\lambda=1$. Let $\underline{w}=1-x$ and $\bar{w}=1+x$. In this example $x$ is a measure of the welfare burden imposed by the requirement of IV. This welfare burden vanishes as $x \rightarrow 0$, implying that $\underline{w} \rightarrow \bar{w}$. As $x \rightarrow \frac{1}{2}$, one converges to the case with $\theta_{L} \bar{w}=\theta_{H} \underline{w}$ which precludes any information aggregation. One may verify that, for sufficiently small $x$, a provision rule with four pooling levels is optimal. For $x \geq 2^{-\frac{3}{2}}$, an optimal provision rule with four pooling levels is transformed into the degenerate case with only three pooling levels. Finally, for $x$ close to $\frac{1}{2}$ a provision rule with only two pooling levels is superior.

\section{Sampling}

In section 2 we observed that in a continuum economy, the problem of information aggregation has no structure because individuals are indifferent about which taste parameter to announce. For the definition of the informative voting problem, we just assumed that individuals break this indifference taking their most preferred perception of the state of the economy into account. The purpose of this section is to derive the $I V$ constraints in a way that avoids this ad-hocery.

We discuss informative voting decisions by a finite random sample of $N$ individuals. In a finite sample, each sample member has a strictly positive

\footnotetext{
${ }^{15}$ To see this, suppose now that here are two provision levels below $\bar{Q}_{L}$. Since preferences in reduced form are single-peaked this individual prefers the larger of these two. But $I V$ rules out this possibility.
} 
influence on the mechanism designer's perception of $p$. This structure can be used to study the limiting case as individual influence becomes arbitrary small. We thus regard the sampling approach to be a way to single out the "reasonable" outcome in a continuum economy. Indeed, as we will show below, as $N \rightarrow \infty$, the optimal provision rule based on sampling converges to the provision rule which solves the informative voting problem $P$.

The sampling procedure that we study is based on the assumption that the cost of public good provision is shared equally among all individuals in the economy. Put differently, we consider an incentive compatible scheme of public goods finance that does not discriminate between individuals who happen to be in the sample and individuals with the same characteristics who are not sample members.

\section{Mechanism design based on sampling}

For the purpose of information aggregation, the mechanism designer communicates with a random sample $S_{N}$ of $N$ individuals. He uses the number $m=\#\left\{i \in S_{N} \mid \theta^{i}=\theta_{H}\right\}$ of individuals with a high taste parameter to update his prior beliefs on the actual state $p$ of the economy. Based on these posterior beliefs he decides on public good provision. Consequently, a provision rule based on sampling of size $N$

$$
Q_{N}:\{0,1, \ldots, N\} \rightarrow \mathbb{R}_{+}, \quad m \mapsto Q_{N}(m),
$$

specifies a quantity of the public good for each possible $m$.

A scheme of public goods finance has to satisfy incentive compatibility if applied to the whole economy and to achieve a balanced budget. Moreover, with respect to their payment obligations, sampled individuals must not be treated differently than individuals who are not in the sample. As a consequence, equal cost sharing is the only admissible payment scheme and preferences can be represented in reduced form,

$$
U(m, \theta, w)=\theta Q_{N}(m)-\frac{K\left(Q_{N}(m)\right)}{w} .
$$

In a revelation game, each sampled individual has an impact on the number $m$ of high taste parameters which are observed by the mechanism designer. The following incentive conditions ensure that each sampled individual is willing to reveal her taste parameter. We call those constraints the informative-voting-under-sampling-of-size- $N\left(I V_{N}\right)$ constraints.

Definition 4 A provision rule $Q_{N}$ allows for informative voting under sampling of size $N\left(I V_{N}\right)$ if the following inequalities hold for all $m \in\{0, \ldots, N-$ $1\}$ and for all $w \in W$ :

$$
\begin{aligned}
& \theta_{L} w Q_{N}(m)-K\left(Q_{N}(m)\right) \geq \theta_{L} w Q_{N}(m+1)-K\left(Q_{N}(m+1)\right), \\
& \theta_{H} w Q_{N}(m)-K\left(Q_{N}(m)\right) \leq \theta_{H} w Q_{N}(m+1)-K\left(Q_{N}(m+1)\right) .
\end{aligned}
$$


The $I V_{N}$ constraints ensure that the truth is a dominant strategy in a game, in which individuals announce either a high or a low taste parameter and preferences are given in reduced form.

We assume that the mechanism designer chooses $Q_{N}$ in order to maximize utilitarian welfare from the ex ante perspective. Ex ante, the sample distribution $m$ and hence the posterior beliefs about the state of the economy are unknown. The mechanism designer takes $m$ to be the realization of a random variable which behaves in accordance with the planner's prior beliefs and the Law of Large Numbers. As shown in the appendix, this implies that the mechanism designer takes $m$ to be the realization of a random variable which is uniformly distributed over the support $\{0,1, \ldots, N\}$. Consequently, an explicit expression for this utilitarian objective function can be derived; see section A.3 of the Appendix.

Lemma 4 Under Assumption 1, a provision rule based on sampling of size $N, Q_{N}$, gives rise to the following level of expected utilitarian welfare

$$
E W_{N}:=\lambda \frac{1}{N+1} \sum_{m=0}^{N}\left\{\bar{v}\left(\frac{m+1}{N+2}\right) Q_{N}(m)-K\left(Q_{N}(m)\right)\right\} .
$$

According to Lemma 4, a mechanism designer who observes a sample in which $m$ individuals have a high taste parameter ends up with an effective valuation of the public good given by

$$
\bar{v}\left(\frac{m+1}{N+2}\right)=\frac{m+1}{N+2} \frac{\theta_{H}}{\lambda}+\frac{N-m+1}{N+2} \frac{\theta_{L}}{\lambda} .
$$

Note that this effective valuation is strictly increasing in $m$, and for all $m \in\{0, \ldots, N\}$, it exceeds $\bar{v}(0)$ and falls short of $\bar{v}(1)$.

Definition 5 The following problem is called the finite sample problem $P_{N}$ : Choose a provision rule based on sampling of size $N, Q_{N}$ in order to maximize $E W_{N}$ subject to the $I V_{N}$ constraints. The solution to this problem is denoted by $Q_{N}^{* *}$, the induced optimal welfare level by $E W_{N}^{* *}$.

\section{Large samples and the Condorcet Jury Theorem}

In the following we will study the behavior of $Q_{N}^{* *}$ and $E W_{N}^{* *}$ as $N \rightarrow \infty$. The main result of this section establishes that as $N \rightarrow \infty$ the informative voting problem $P$ and the finite sample problem $P_{N}$ are essentially equivalent.

Proposition 3 As $N \rightarrow \infty$, the welfare level which is realized under a solution of the finite sample problem $P_{N}, E W_{N}^{* *}$, converges to the welfare 
level which is realized under a solution of the informative voting problem $P$, $E W^{* *}$. Formally,

$$
\lim _{N \rightarrow \infty} E W_{N}^{* *}=E W^{* *} .
$$

Moreover, if $Q^{* *}$ is not constant, ${ }^{16}$ then for any $N \in \mathbb{N}, E W_{N}^{* *}<E W^{* *}$.

A proof can be found in section A.2 of the appendix. Moreover, the appendix also contains a corollary which strengthens the equivalence between the concepts of informative voting under sampling and informative voting established in Proposition 3: If there is a unique solution $Q^{* *}$ of problem $P$, then the optimal provision rule under $I V$ constraints, $Q^{* *}$, and the optimal provision rule under $I V_{N}$ constraints $Q_{N}^{* *}$ "coincide" in the limit.

As a further corollary of Proposition 3, we can prove a version of the famous Condorcet Jury Theorem. This theorem is concerned with decision making in committees. In its most simple version, ${ }^{17}$ the theorem says that whenever each committee member has some private information on the state of the world and, moreover, all committee members have identical preferences, then it is always preferable to have a larger committee. The underlying logic is that a larger committee has more pieces of information available and is thus more likely to take the "right" decision.

For our version of the Condorcet Jury Theorem we interpret the random sample $S_{N}$ as a committee. ${ }^{18}$ We then ask whether there is a finite optimal sample size.

Corollary 1 Suppose that provision rule $Q^{* *}$ is not constant. Then for any given $N \in \mathbb{N}$ there exists $N^{\prime} \in \mathbb{N}$ with $N^{\prime}>N$ such that $E W_{N}^{* *}<E W_{N^{\prime}}^{* *}$.

Whenever some degree of information aggregation is desirable, there is no optimal sample size. The intuition for this result is the following. A growing sample size $N$ implies that the mechanism designer's estimate of the actual state of the economy becomes more precise. This allows for a better adjustment of the final provision level to the actual state of the economy. However, a larger $N$ also implies a larger set of $I V_{N}$ constraints. These additional constraints, however, do not undermine this reasoning. A mechanism designer with a large sample can always mimic a small sample outcome by choosing not to use certain pieces of information. Hence, larger samples generate additional degrees of freedom for the mechanism designer.

\footnotetext{
${ }^{16}$ Sufficient conditions are: $\theta_{L} \bar{w} \leq \theta_{H} \underline{w}, \hat{Q}^{*}(0) \leq Q^{*}(1)$ and $Q^{*}(0) \leq \tilde{Q}^{*}(1)$.

${ }^{17}$ See Austen-Smith and Banks (1996) and Piketty (1999). A more advanced treatment can be found in Feddersen and Pesendorfer (1997).

${ }^{18}$ A similar approach can be found in Auriol and Gary-Bobo (2005).
} 


\section{Concluding Remarks}

We have addressed a problem of public goods provision in a continuum economy with uncertainty about the average valuation of the public good. We have shown that in this environment any anonymous allocation mechanism faces a problem of equilibrium selection, since individuals are literally indifferent between all actions they have available.

We have formulated two different approaches for dealing with this problem. The first, rather naive, idea is to use an individual's preference over the composition of the economy to break his indifference; i.e. whenever he is indifferent between, say, announcements $a$ and $b$ but would be happy if more individuals in the economy announced $b$, then assume that the individual in question will announce $b$ as well. ${ }^{19}$

The second approach, informative voting under sampling, distinguishes more explicitly between information aggregation to determine the optimal quantity of a public good and the financing of this desired quantity. A scheme of public goods finance that has to be incentive compatible for the whole economy is taken as given. Under this premise, a large random sample of individuals is used to aggregate information. Sampled individuals now have an impact on public good provision, and this unambiguously governs their behavior. Hence, the multiple equilibrium problem is eliminated.

Finally we have shown, that, for large random samples, these two different approaches are equivalent. That is, the simple rule which we refer to as informative voting can be interpreted as the limit outcome of vanishing individual influence under a voting mechanism with a finite number of individuals.

A third approach that also provides a foundation of the informative voting constraints can be found in Bierbrauer (2005a). In this paper an admissible provision rule for public goods has to satisfy a condition of collective incentive compatibility, which eliminates incentives for collective manipulations of the perceived state of the economy. For the quasi-linear environment analyzed in the present paper, this requirement is shown to be equivalent to the informative voting constraints.

\footnotetext{
${ }^{19}$ In models with voting over two alternatives and a continuum of voters one often finds the statement that this behavior is the only one which survives the elimination of weakly dominated strategies. Implicitly, this reasoning appeals to a large but finite economy. Examples include Gersbach (2005) or Meirowitz (2005).
} 


\section{A Appendix}

\section{A.1 Proof of Proposition 2}

Before turning to the proof of the proposition we state the formal definition of a provision rule with two, three and four mass points, respectively.

We first need to introduce the following piece of notation. For a provision level $Q$ with $Q<\bar{Q}_{L}$, denote by $\hat{Q}$ the provision level which satisfies $Q<\hat{Q}$ and $\theta_{L} \bar{w} Q-K(Q)=\theta_{L} \bar{w} \hat{Q}-K(\hat{Q})$; that is, an individual with effective valuation $\theta_{L} \bar{w}$ is indifferent between the provision levels $Q$ and $\hat{Q}$. Likewise, for any $Q$ with $\underline{Q}_{H}<Q$, denote by $\tilde{Q}$ the provision level with $\tilde{Q}<Q$ and $\theta_{H} \underline{w} Q-K(Q)=\theta_{H} \underline{w} \tilde{Q}-K(\tilde{Q})$.

Definition 6 An increasing provision rule $Q_{4}: p \mapsto Q_{4}(p)$ is said to have four pooling levels if

$$
Q_{4}(p):= \begin{cases}Q_{4}^{s} & \text { for } 0 \leq p \leq \hat{p} \\ \hat{Q}_{4}^{s} & \text { for } \quad \hat{p}<p<\hat{p}^{\prime} \\ Q^{*}(p) & \text { for } \quad \hat{p}^{\prime} \leq p \leq \tilde{p}^{\prime} \\ \tilde{Q}_{4}^{l} & \text { for } \quad \tilde{p}^{\prime}<p<\tilde{p} \\ Q_{4}^{l} & \text { for } \quad \tilde{p} \leq p \leq 1\end{cases}
$$

where the critical indices are implicitly defined by the following equations: ${ }^{20}$

$$
\bar{v}(\hat{p})=\theta_{L} \bar{w}, \quad Q^{*}\left(\hat{p}^{\prime}\right)=\hat{Q}_{4}^{s}, \quad Q^{*}\left(\tilde{p}^{\prime}\right)=\tilde{Q}_{4}^{l}, \quad \bar{v}(\tilde{p})=\theta_{H} \underline{w} .
$$

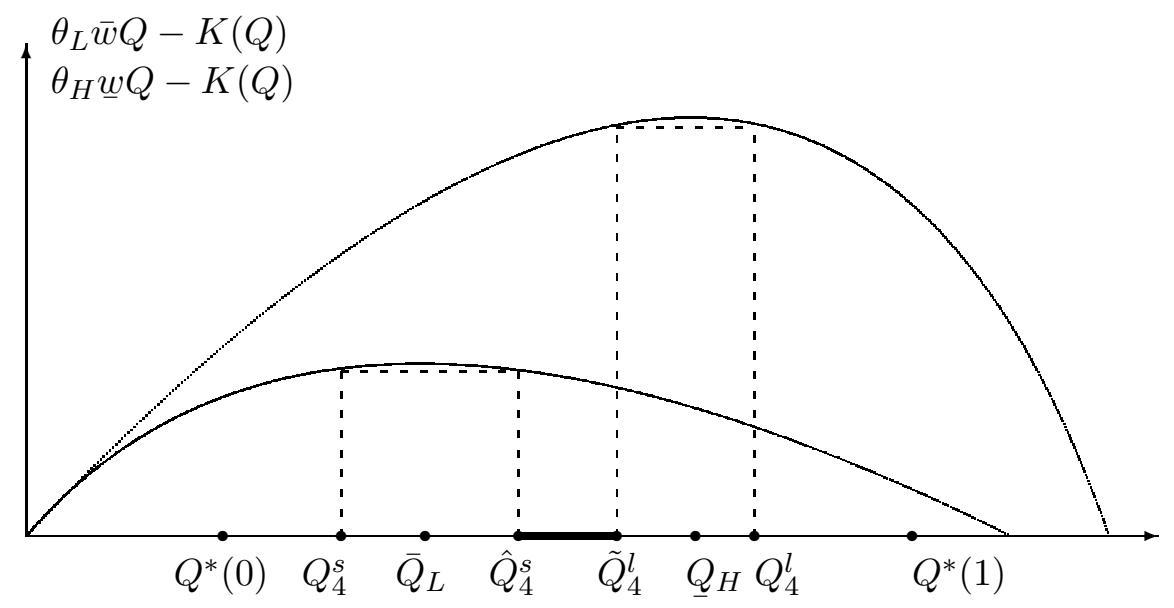

Figure 3: The figure depicts a provision rule characterized by four pooling levels.

\footnotetext{
${ }^{20}$ This definition already presumes an optimal choice of the critical indices: a utilitarian planner will choose, e.g. , $\hat{p}$ according to the following criterion: Let $Q(p)=Q_{4}^{s}$ if and only if $\bar{v}(p) Q_{4}^{s}-K\left(Q_{4}^{s}\right)$ exceeds $\bar{v}(p) \hat{Q}_{4}^{s}-K\left(\hat{Q}_{4}^{s}\right)$. Given the binding IV constraint which links $Q_{4}^{s}$ and $\hat{Q}_{4}^{s}$, this is equivalent to $Q(p)=Q_{4}^{s}$ if and only if $\bar{v}(p) \leq \theta_{L} \bar{w}$.
} 
If the range where the provision rules $Q_{4}$ and $Q^{*}$ coincide shrinks to a singleton one has a provision rule characterized by three pooling levels.

Definition 7 An increasing provision rule $Q_{3}: p \mapsto Q_{3}(p)$ is said to have three pooling levels if

$$
Q_{3}(p):= \begin{cases}Q_{3}^{s} & \text { for } \quad 0 \leq p \leq \hat{p} \\ Q_{3}^{m} & \text { for } \quad \hat{p}<p<\tilde{p} \\ Q_{3}^{l} & \text { for } \quad \tilde{p} \leq p \leq 1\end{cases}
$$

where the critical indices $\hat{p}$ and $\tilde{p}$ are defined implicitly by the equations

$$
\bar{v}(\hat{p})=\theta_{L} \bar{w} \quad \text { and } \quad \bar{v}(\tilde{p})=\theta_{H} \underline{w} .
$$

The medium sized provision level $Q_{3}^{m}$ is now linked via a binding $I V$ constraint with $Q_{3}^{s}$ and via a binding $I V$ constraint with $Q_{3}^{l}$, i.e. $Q_{3}^{m}=\hat{Q}_{3}^{s}=\tilde{Q}_{3}^{l}$. If the medium range shrinks further, there is no provision level lying between $\bar{Q}_{L}$ and $\underline{Q}_{H}$. Such a provision rule is characterized by two pooling levels.

Definition 8 An increasing provision rule $Q_{2}: p \mapsto Q_{2}(p)$ is said to have two pooling levels if

$$
Q_{2}(p):= \begin{cases}Q_{2}^{s} & \text { for } 0 \leq p \leq \hat{p} \\ Q_{2}^{l} & \text { for } \quad \hat{p}<p \leq 1\end{cases}
$$

where the critical index $\hat{p}$ is defined implicitly by the equation

$$
\bar{v}(\hat{p}) Q_{2}^{s}-K\left(Q_{2}^{s}\right)=\bar{v}(\hat{p}) Q_{2}^{l}-K\left(Q_{2}^{l}\right) .
$$

Proof of Proposition 2. The required arguments are lengthy but not difficult. Hence the arguments are only sketched. As has been shown in Proposition 1, there are parameter constellations such that $Q^{* *}$ is indeed constant. Now suppose that $Q^{* *}$ is not constant. Thus, by Proposition 1, $\theta_{L} \bar{w} \leq \theta_{H} \underline{w}$ and hence $Q^{*}(0) \leq \bar{Q}_{L} \leq \underline{Q}_{H} \leq Q^{*}(1)$. We show within the next three steps that $Q^{* *}$ is a provision rule with either two, three or four pooling levels.

Step 1. Denote by $\mathcal{V}_{Q}$ the image of a provision rule $Q$, i.e. $x \in \mathcal{V}_{Q}$ if and only if there exists $p \in[0,1]$ with $Q(p)=x$. Under the IV constraints, there exists at most one element $x \in \mathcal{V}_{Q}$ with $x<\bar{Q}_{L}$.

Proof of step 1. To see this, suppose, to the contrary, that there exist 
$x, y \in \mathcal{V}_{Q}$ with $x<y<\bar{Q}_{L}$. Under IV, as characterized in Lemma 3 , this implies that there exist $p$ and $p^{\prime}>p$, with $Q(p)<Q\left(p^{\prime}\right)<\bar{Q}_{L}$. This yields

$$
\theta_{L} \bar{w} Q(p)-K(Q(p))<\theta_{L} \bar{w} Q\left(p^{\prime}\right)-K\left(Q\left(p^{\prime}\right)\right),
$$

a contradiction to the IV requirement for an individual with effective valuation $\theta_{L} \bar{w}$. Analogously one shows that the image of an admissible provision rule contains at most one element $x$ with $x>Q_{H}$.

Step 2. We now show that a provision rule $Q$ for which there exists $y \in \mathcal{V}_{Q}$ with $y \in\left[\bar{Q}_{L}, Q_{H}\right]$ is a candidate for a solution only if there exist $x, z \in \mathcal{V}_{Q}$ with $x<\bar{Q}_{L}$ and $\underline{Q}_{H}<z$.

Proof of step 2. To this end, we first argue that a provision rule $Q$ for which there exist neither $x \in \mathcal{V}_{Q}$ with $x<\bar{Q}_{L}$ nor $z \in \mathcal{V}_{Q}$ with $z>\underline{Q}_{H}$ cannot be optimal. Such a hypothetical provision rule would satisfy $\mathcal{V}_{Q} \subset\left[\bar{Q}_{L}, \underline{Q}_{H}\right]$. This can be optimal only if $\mathcal{V}_{Q}=\left[\bar{Q}_{L}, Q_{H}\right]$, which would be the degenerate case of a provision rule with four pooling levels, which results as the limit outcome as $Q_{4}^{s}$ converges to $\bar{Q}_{L}$ and $Q_{4}^{l}$ converges to $\underline{Q}_{H}$. Under a provision rule characterized by four pooling levels, expected welfare $E W$ satisfies the following equation:

$$
\begin{aligned}
\frac{E W}{\lambda}= & \hat{p}\left[\bar{v}\left(\frac{\hat{p}}{2}\right) Q_{4}^{s}-K\left(Q_{4}^{s}\right)\right]+\left(\hat{p}^{\prime}-\hat{p}\right)\left[\bar{v}\left(\frac{\hat{p}^{\prime}+\hat{p}}{2}\right) \hat{Q}_{4}^{s}-K\left(\hat{Q}_{4}^{s}\right)\right] \\
& +\int_{\hat{p}^{\prime}}^{\tilde{p}^{\prime}}\left\{\bar{v}(p) Q^{*}(p)-K\left(Q^{*}(p)\right)\right\} d p+\left(\tilde{p}-\tilde{p}^{\prime}\right)\left[\bar{v}\left(\frac{\tilde{p}^{\prime}+\tilde{p}}{2}\right) \tilde{Q}_{4}^{l}-K\left(\tilde{Q}_{4}^{l}\right)\right] \\
& +(1-\tilde{p})\left[\bar{v}\left(\frac{1+\tilde{p}}{2}\right) Q_{4}^{l}-K\left(Q_{4}^{l}\right)\right],
\end{aligned}
$$

where $\hat{Q}_{4}^{s}$ and $\hat{p}^{\prime}$ are implicit functions of $Q_{4}^{s}$. Similarly, $\tilde{Q}_{4}^{l}$ and $\tilde{p}^{\prime}$ are implicit functions of $Q_{4}^{l}$. Taking these functional relationships into account, one may compute the partial derivatives and verify that

$$
\lim _{Q_{4}^{s} \rightarrow \bar{Q}_{L}} \frac{\partial E W\left(Q_{4}^{s}, Q_{4}^{l}\right)}{\partial Q_{4}^{s}}<0 \quad \text { and } \quad \lim _{Q_{4}^{l} \rightarrow \underline{Q}_{H}} \frac{\partial E W\left(Q_{4}^{s}, Q_{4}^{l}\right)}{\partial Q_{4}^{l}}>0
$$

Thus, $Q_{4}^{s}=\bar{Q}_{L}$ and $Q_{4}^{l}=Q_{H}$ cannot be optimal.

We now argue in a similar manner that it cannot be optimal to choose a provision rule with $y, z \in \mathcal{V}_{Q}$ satisfying $\bar{Q}_{L}<y<\underline{Q}_{H}<z$, but without $x \in \mathcal{V}_{Q}$ satisfying $x<\bar{Q}_{L}$ :

Define $\tilde{z}<\underline{Q}_{H}$ by the equation $\theta_{H} \underline{w} z-K(z)=\theta_{H} \underline{w} \tilde{z}-K(\tilde{z})$. Note that for such a provision rule to be a optimal under $I V$ it has to be true that $y \leq \tilde{z}$ and that $\mathcal{V}_{Q}=\left[\bar{Q}_{L}, \tilde{z}\right] \cup\{z\}$ by step 1 and the requirement of optimality. Again, this is a degenerate case of a provision rule with four pooling levels , namely the one that results as $Q_{4}^{s}$ converges to $\bar{Q}_{L}$ and $Q_{4}^{l}=z$. As above, 
this hypothetical solution can be ruled out as

$$
\lim _{Q_{4}^{s} \rightarrow \bar{Q}_{L}} \frac{\partial E W\left(Q_{4}^{s}, Q_{4}^{l}\right)}{\partial Q_{4}^{s}}<0 .
$$

The analogous argument allows us to rule out a provision rule with $x, y \in \mathcal{V}_{Q}$ and $x<\bar{Q}_{L}<y<\underline{Q}_{H}$ but without $z \in \mathcal{V}_{Q}$ satisfying $z>\underline{Q}_{H}$.

Step 3. We now claim that a provision rule, for which there exist $x, y \in \mathcal{V}_{Q}$ with $\bar{Q}_{L}<x<y<\underline{Q}_{H}$, is a candidate for a solution only if the whole interval satisfies $[x, y] \subset \mathcal{V}_{Q}$.

Proof of Step 3. By step 2, there are $a, b \in \mathcal{V}_{Q}$ with $a<\bar{Q}_{L}<\underline{Q}_{H}<b$. Define $\tilde{b}<\underline{Q}_{H}$ by the equation $\theta_{H} \underline{w} b-K(b)=\theta_{H} \underline{w} \tilde{b}-K(\tilde{b})$. Analogously, define $\hat{a}>\bar{Q}_{L}$ by $\theta_{L} \bar{w} a-K(a)=\theta_{L} \bar{w} \hat{a}-K(\hat{a})$. For the hypothesized provision rule to be a optimal under $I V$, it has to be true that $\hat{a} \leq x<y \leq \tilde{b}$ and that $[x, y] \subset[\hat{a}, \tilde{b}] \subset \mathcal{V}_{Q}$.

Steps 1-3 imply that an optimal provision rule under $I V$, which is not constant, has to be one with two, three or four pooling levels.

\section{A.2 Proof of Proposition 3}

We start with the observation that the maximal welfare level $E W^{* *}$, which is achievable under $I V$ constraints, is, for any sample size $N$, an upper bound for the expected welfare that is achievable under $I V_{N}$ constraints. To establish this claim, we define, for any given $N \in \mathbb{N}$, the following piecewise constant continuation of the solution of problem $P_{N}$, which we denote by $\tilde{Q}_{N}^{* *}$ :

$$
\begin{aligned}
\tilde{Q}_{N}^{* *}:[0,1] & \rightarrow\left\{Q_{N}^{* *}(m)\right\}_{m=0}^{N}, \quad \text { with } \\
\tilde{Q}_{N}^{* *}(p) & :=Q_{N}^{* *}(m) \text { for } \frac{m}{N+1} \leq p<\frac{m+1}{N+1}, \\
\tilde{Q}_{N}^{* *}(1) & :=Q_{N}^{* *}(N) .
\end{aligned}
$$

The welfare level induced by $\tilde{Q}_{N}^{* *}$ is denoted $\widetilde{E W}_{N}^{* *}$.

Lemma 5 For any $N \in \mathbb{N}$, the following inequalities hold:

$$
E W_{N}^{* *} \leq \widetilde{E W}_{N}^{* *} \leq E W^{* *} .
$$

The first inequality is strict if and only if $Q_{N}^{* *}$ is not constant.

Proof of Lemma 5. We note that the requirement of $I V_{N}$ can be characterized analogous to the characterization of the $I V$ property in Lemma 
3. Accordingly, a provision rule based on sampling $Q_{N}$ satisfies $I V_{N}$ if and only if the following two properties hold for any pair $m, m^{\prime} \in\{0,1, \ldots, N\}$ with $m^{\prime}>m$ :

i) $Q_{N}$ is increasing: $Q_{N}\left(m^{\prime}\right) \geq Q_{N}(m)$.

ii) If $Q_{N}\left(m^{\prime}\right)>Q_{N}(m)$, then

$$
\theta_{H} \underline{w} \geq \frac{K\left(Q_{N}\left(m^{\prime}\right)\right)-K\left(Q_{N}(m)\right)}{Q_{N}\left(m^{\prime}\right)-Q_{N}(m)} \geq \theta_{L} \bar{w} .
$$

By definition, $\tilde{Q}_{N}^{* *}$ is monotonically increasing in $p$ and inherits the $I V$ property from the fact that $\left\{Q_{N}^{* *}(m)\right\}_{m=0}^{N}$ is $I V_{N}$. This is obvious from the above characterization of $I V_{N}$ and Lemma 3. Hence, by the optimality of $Q^{* *}$ among the provision rules satisfying IV, $\widetilde{E W}_{N}^{* *} \leq E W^{* *}$. It thus remains to be shown that $E W_{N}^{* *} \leq \widetilde{E W}_{N}^{* *}$.

In order to compute $\widetilde{E W}_{N}^{* *}$, we first collect a number of observations which are easily verified by the reader.

1. For all $\underline{p}, \bar{p} \in[0,1]$

$$
\int_{\underline{p}}^{\bar{p}} \bar{v}(p) d p=(\bar{p}-\underline{p}) \bar{v}\left(\frac{\bar{p}+\underline{\underline{p}}}{2}\right) .
$$

2. For all $m \in\{0,1, \ldots, N\}$

$$
\frac{m+\frac{1}{2}}{N+1}=\frac{m+1}{N+2}+\frac{m-\frac{1}{2} N}{(N+1)(N+2)} .
$$

3. For all $x, y \in[0,1]$ with $x+y \in[0,1], \bar{v}(x+y)=\bar{v}(x)+\frac{\theta_{H}-\theta_{L}}{\lambda} y$.

4. By definition of $E W_{N}^{* *}$ and $Q_{N}^{* *}$,

$$
E W_{N}^{* *}=\lambda \frac{1}{N+1} \sum_{m=0}^{N}\left\{\bar{v}\left(\frac{m+1}{N+2}\right) Q_{N}^{* *}(m)-K\left(Q_{N}^{* *}(m)\right)\right\} .
$$

Using these equalities, one arrives at

$$
\begin{aligned}
\widetilde{E W}_{N}^{* *} & =\lambda \int_{0}^{1}\left\{\bar{v}(p) \tilde{Q}_{N}^{* *}(p)-K\left(\tilde{Q}_{N}^{* *}(p)\right)\right\} d p \\
& =E W_{N}^{* *}+\frac{\theta_{H}-\theta_{L}}{(N+1)^{2}(N+2)} \sum_{m=0}^{N}\left(m-\frac{1}{2} N\right) Q_{N}^{* *}(m) .
\end{aligned}
$$

To complete the proof, we show that $\sum_{m=0}^{N}\left(m-\frac{1}{2} N\right) Q_{N}^{* *}(m) \geq 0$. This expression equals

$$
\sum_{m=0}^{\frac{1}{2} N}\left(\frac{1}{2} N-m\right)\left(Q_{N}^{* *}(N-m)-Q_{N}^{* *}(m)\right)
$$


if $N$ is even and

$$
\sum_{m=0}^{\frac{N-1}{2}}\left(\frac{1}{2} N-m\right)\left(Q_{N}^{* *}(N-m)-Q_{N}^{* *}(m)\right)
$$

if $N$ is odd. However, as $Q_{N}^{* *}$ is increasing, those sums are non-negative. Moreover, they are strictly positive, and hence $E W_{N}^{* *}<E \tilde{W}_{N}^{* *}$ if and only if $Q_{N}^{* *}$ is not constant.

Proof of the first statement of Proposition 3. We now establish that $E W_{N}^{* *}$ converges to $E W^{* *}$ as $N$ goes to $\infty$.

Let $Q^{* *}$ be a solution of problem $P$ and $Q_{\mid N}^{* *}$ its restriction to the domain $\{0,1, \ldots, N\}$. Formally, for each $m \in\{0,1, \ldots, N\}, Q_{\mid N}^{* *}(m)$ is defined by the equation $Q_{\mid N}^{* *}(m):=Q^{* *}\left(\frac{m}{N}\right)$. One easily verifies that $Q_{\mid N}^{* *}$ has the $I V_{N}$ property.

Denote by $E W_{\mid N}^{* *}$ the expected welfare level induced by $Q_{\mid N}^{* *}$. Then, since $Q_{N}^{* *}$ is optimal among the provision rules with the $I V_{N}$ property, $E W_{\mid N}^{* *} \leq E W_{N}^{* *}$. Moreover,

$$
\begin{aligned}
E W_{\mid N}^{* *}= & \lambda \frac{1}{N+1} \sum_{m=0}^{N}\left\{\bar{v}\left(\frac{m+1}{N+2}\right) Q^{* *}\left(\frac{m}{N}\right)-K\left(Q^{* *}\left(\frac{m}{N}\right)\right)\right\} \\
= & \lambda \frac{1}{N+1} \sum_{m=0}^{N}\left\{\bar{v}\left(\frac{m}{N}\right) Q^{* *}\left(\frac{m}{N}\right)-K\left(Q^{* *}\left(\frac{m}{N}\right)\right)\right\} \\
& +\frac{\theta_{H}-\theta_{L}}{N(N+1)(N+2)} \sum_{m=0}^{N}(N-2 m) Q^{* *}\left(\frac{m}{N}\right) .
\end{aligned}
$$

The first term in this sum is known as the Riemann $\operatorname{sum}^{21}$ for $\bar{v}(p) Q^{* *}(p)-$ $K\left(Q^{* *}(p)\right.$ and thus converges to $E W^{* *}$ for growing $N$. The second term in the sum is bounded from above by the expression $\frac{\theta_{H}-\theta_{L}}{N+2} Q^{* *}(1)$, which vanishes as $N \rightarrow \infty$. Consequently,

$$
\lim _{N \rightarrow \infty} E W_{\mid N}^{* *}=E W^{* *} .
$$

Summing up and using Lemma 5, we have

$$
E W^{* *}=\lim _{N \rightarrow \infty} E W_{\mid N}^{* *} \leq \lim _{N \rightarrow \infty} E W_{N}^{* *} \leq E W^{* *} .
$$

\footnotetext{
${ }^{21}$ See e.g. Heuser (1998, Ch.10).
} 
Proof of the second statement of Proposition 3. We now show that $E W_{N}^{* *}$ is strictly smaller than $E W^{* *}$ for any $N$ provided that $Q^{* *}$ is not a constant provision rule.

Denote by $E W^{u}$ the maximal level of expected welfare which can be generated by some constant provision rule. If the solution to problem $P$ is not constant, then $E W^{u}<E W^{* *}$. If, for $N \in \mathbb{N}$, a solution to problem $P_{N}$ is constant, then $E W_{N}^{* *}=E W^{u}<E W^{* *}$. If, by contrast, $Q_{N}^{* *}$ is not constant, then $E W_{N}^{* *}<E W^{* *}$ by Lemma 5 .

We finally establish the following corollary of Proposition 3.

Corollary 2 Suppose there is a unique solution $Q^{* *}$ to problem $P$, and let $\tilde{Q}_{N}^{* *}$ be defined as in $(2)$. Then, for all $p \in[0,1]$

$$
\lim _{N \rightarrow \infty} \tilde{Q}_{N}^{* *}(p)=Q^{* *}(p) .
$$

Proof of Corollary 2. By Lemma 5 and Proposition 3,

$$
\lim _{N \rightarrow \infty} E W_{N}^{* *}=\lim _{N \rightarrow \infty} \widetilde{E W}_{N}^{* *}=E W^{* *} .
$$

$\tilde{Q}_{N}^{* *}$ is $I V_{N}$ for all $N \in \mathbb{N}$. Thus, the uniqueness of $Q^{* *}$ among the provision rules which satisfy $I V$ and yield welfare level $E W^{* *}$ implies the claimed property of pointwise convergence.

\section{A.3 Further Proofs}

Proof of Lemma 3. We first show that the $I V$ constraints imply statements i) and ii). The $I V$ constraints imply that the following two inequalities have to hold,

$$
\begin{aligned}
& \theta_{L} w Q(p)-K(Q(p)) \geq \theta_{L} w Q\left(p^{\prime}\right)-K\left(Q\left(p^{\prime}\right)\right), \\
& \theta_{H} w Q(p)-K(Q(p)) \leq \theta_{H} w Q\left(p^{\prime}\right)-K\left(Q\left(p^{\prime}\right)\right) .
\end{aligned}
$$

Adding up these inequalities yields:

$$
\left(\theta_{H}-\theta_{L}\right) w\left[Q\left(p^{\prime}\right)-Q(p)\right] \geq 0
$$


This establishes i). Now suppose that $Q(p)<Q\left(p^{\prime}\right)$. Then for any $w \in W$, $I V$ requires that

$$
\frac{K\left(Q\left(p^{\prime}\right)\right)-K(Q(p))}{Q\left(p^{\prime}\right)-Q(p)} \geq \theta_{L} w
$$

This property holds for all $w \in W$ if and only if it holds for the largest skill level $\bar{w}$,

$$
\frac{K\left(Q\left(p^{\prime}\right)\right)-K(Q(p))}{Q\left(p^{\prime}\right)-Q(p)} \geq \theta_{L} \bar{w} .
$$

Likewise we derive the requirement

$$
\theta_{H} \underline{w} \geq \frac{K\left(Q\left(p^{\prime}\right)\right)-K(Q(p))}{Q\left(p^{\prime}\right)-Q(p)}
$$

This establishes ii).

The proof that i) and ii) imply that the $I V$ property holds is now immediate.

Proof of Proposition 1. Statement i) is a direct consequence of Lemma 3. It thus remains to be shown that $Q^{*}$ satisfies $I V$ if and only if $\underline{w}=\bar{w}$. To prove the "only if" part, suppose that $\underline{w} \neq \bar{w}$. Consider the indirect utility function $U^{*}(p, \theta, w)$. As shown in the body of the text, $U^{*}$ is increasing in $p$ as long as $\theta w>\bar{v}(p)$, i.e. the individual's effective valuation of the public good exceeds the effective utilitarian valuation. Analogously, $U^{*}$ is decreasing in $p$ if $\theta w$ falls short of the utilitarian valuation. Now consider a level of $p$ such that ${ }^{22}$

$$
\theta_{L} \bar{w}>\bar{v}(p)>\frac{\theta_{L}}{\lambda}
$$

This implies that there exists a critical value $\hat{w} \in] \underline{w}, \bar{w}[$ such that all individuals with $\theta^{i}=\theta_{L}$ and $w^{i}>\hat{w}$ have an effective valuation $\theta_{L} w^{i}$ exceeding $\bar{v}(p)$. Therefore, they would prefer a slightly larger perceived value of $p$. This violates the $I V$ property.

To prove the "if" part, suppose that $\underline{w}=\bar{w}=: \tilde{w}$. As $Q^{*}$ is a strictly increasing function, Lemma 3 states that $Q^{*}$ satisfies $I V$ if and only if $p^{\prime}>p$ implies that

$$
\theta_{H} \tilde{w} \geq \frac{K\left(Q^{*}\left(p^{\prime}\right)\right)-K\left(Q^{*}(p)\right)}{Q^{*}\left(p^{\prime}\right)-Q^{*}(p)} \geq \theta_{L} \tilde{w}
$$

\footnotetext{
${ }^{22}$ As $\bar{v}(p)$ is a convex combination of $\frac{\theta_{H}}{\lambda}$ and $\frac{\theta_{L}}{\lambda}$, for any $x \in\left[\frac{\theta_{L}}{\lambda}, \frac{\theta_{H}}{\lambda}\right]$ there exists $p$ such that $\bar{v}(p)=x$.
} 
We show in the following that the convexity of $K$ and the first order conditions characterizing $Q^{*}$ imply that these inequalities are indeed satisfied for any pair $p^{\prime}$ and $p$ with $p^{\prime}>p$. From the convexity of the cost function, we have

$$
K^{\prime}\left(Q^{*}\left(p^{\prime}\right)\right)>\frac{K\left(Q^{*}\left(p^{\prime}\right)\right)-K\left(Q^{*}(p)\right)}{Q^{*}\left(p^{\prime}\right)-Q^{*}(p)}>K^{\prime}\left(Q^{*}(p)\right) .
$$

With $\underline{w}=\bar{w}=: \tilde{w}$, the first order conditions characterizing $Q^{*}$ imply

$$
\begin{aligned}
& K^{\prime}\left(Q^{*}(p)\right)=\bar{v}(p)=\tilde{w}\left(p \theta_{H}+(1-p) \theta_{L}\right) \geq \tilde{w} \theta_{L}, \\
& K^{\prime}\left(Q^{*}\left(p^{\prime}\right)\right)=\bar{v}\left(p^{\prime}\right)=\tilde{w}\left(p^{\prime} \theta_{H}+\left(1-p^{\prime}\right) \theta_{L}\right) \leq \tilde{w} \theta_{H} .
\end{aligned}
$$

\section{Proof of Lemma 4.}

Step 1. We first derive the posterior beliefs of the mechanism designer. The mechanism designer's prior beliefs are given by the density function $\phi$. Under Assumption 1, $\phi(p)=1$ for all $p \in[0,1]$. Let $\nu$ be the number of agents with a high taste parameter in a sample of size $N$. Again, from an ex ante perspective, $\nu$ is a random variable, with ${ }^{23}$

$$
\begin{aligned}
\operatorname{pr}(\nu=m) & =\int_{0}^{1} \operatorname{pr}(\nu=m \mid p) \phi(p) d p \\
& =\int_{0}^{1}\left(\begin{array}{c}
N \\
m
\end{array}\right) p^{m}(1-p)^{N-m} d p=\frac{1}{N+1} .
\end{aligned}
$$

This is intuitive, with $p$ uniformly distributed, all possible realizations of $\nu$ are equally likely. Now suppose that $\nu=m$ and consider the conditional density $\phi_{N}$ thereby induced over $p$. By Bayes' rule

$$
\phi_{N}(p \mid \nu=m)=\frac{\operatorname{pr}(\nu=m \mid p) \phi(p)}{\operatorname{pr}(\nu=m)}=(N+1)\left(\begin{array}{l}
N \\
m
\end{array}\right) p^{m}(1-p)^{N-m} .
$$

\footnotetext{
${ }^{23}$ The following relation is used repeatedly:

$$
\int_{0}^{1} p^{m}(1-p)^{N-m} d p=\frac{m !(N-m) !}{(N+1) !} .
$$
}


Step 2. Expected welfare at the interim stage is hence given by

$$
\begin{aligned}
E W_{N}^{i n t}(m) & =\lambda \mathrm{E}\left[\bar{v}(p) Q_{N}(m)-K\left(Q_{N}(m)\right) \mid m\right] \\
& =\lambda \int_{0}^{1}\left[\bar{v}(p) Q_{N}(m)-K\left(Q_{N}(m)\right)\right] \phi(p \mid \nu=m) d p \\
& =\lambda(N+1)\left(\begin{array}{l}
N \\
m
\end{array}\right)\left(\int_{0}^{1}\left[\frac{p \theta_{H}+(1-p) \theta_{L}}{\lambda} Q_{N}(m)-K\left(Q_{N}(m)\right)\right] \times\right. \\
& \left.p^{m}(1-p)^{N-m} d p\right) \\
= & \lambda\left[\frac{m+1}{N+2} \frac{\theta_{H}}{\lambda}+\frac{N-m+1}{N+2} \frac{\theta_{L}}{\lambda}\right] Q_{N}(m)-K\left(Q_{N}(m)\right) .
\end{aligned}
$$

Step 3. From the ex-ante perspective, the outcome $m$ of the sampling procedure is the realization of a random variable, which we denote by $\nu$. Taking expectations over $m$, using (3), expected welfare from the ex-ante perspective equals

$$
\begin{aligned}
E W_{N} & =\sum_{m=0}^{N} E W^{i n t}(m) \operatorname{pr}(\nu=m) \\
& =\lambda \frac{1}{N+1} \sum_{m=0}^{N}\left\{\bar{v}\left(\frac{m+1}{N+2}\right) Q_{N}(m)-K\left(Q_{N}(m)\right)\right\} .
\end{aligned}
$$

\section{References}

Al-Najjar, N. (2004). Aggregation and the law of large numbers in large economies. Games and Economic Behavior, 47:1-35.

Alòs-Ferrer, C. (2002). Individual randomness in economic models with a continuum of agents. Working Paper 9807, Dpt. of Economics, University of Vienna.

Auriol, E. and Gary-Bobo, R. (2005). On robust constitution design. Mimeo, Universit Paris 1 Panthon-Sorbonne.

Austen-Smith, D. and Banks, J. (1996). Information aggregation, rationality and the Condorcet jury theorem. American Political Science Review, 90:34-45.

Bergemann, D. and Morris, S. (2005). Robust mechanism design. Econometrica, 73:1771-1813. 
Bierbrauer, F. (2005a). Collectively incentive compatible tax systems. Mimeo, Max Planck Institute for Research on Collective Goods, Bonn.

Bierbrauer, F. (2005b). Distortionary taxation and the free-rider problem. Mimeo, Max Planck Institute for Research on Collective Goods, Bonn.

Bierbrauer, F. (2005c). Optimal income taxation and public good provision in a two-class economy. Preprint 2005/25, Max Planck Institute for Research on Collective Goods, Bonn.

Chung, K. and Ely, J. (2004). Foundations of dominant strategy mechanisms. Mimeo, Northwestern University.

Clarke, E. (1971). Multipart pricing of public goods. Public Choice, 11:1733 .

Crawford, V. and Sobel, J. (1982). Strategic information transmission. Econometrica, 50:1431-1451.

d'Aspremont, C. and Gérard-Varet, L. (1979). Incentives and incomplete information. Journal of Public Economics, 11:25-45.

Feddersen, T. and Pesendorfer, W. (1997). Voting behaviour and information aggregation in elections with private information. Econometrica, 65:1029-1058.

Feldman, M. and Gilles, C. (1985). An expository note on individual risk without aggregate uncertainty. Journal of Economic Theory, 35:26-32.

Gary-Bobo, R. and Jaaidane, V. (2000). Polling mechanisms and the demand revelation problem. Journal of Public Economics, 76:203-238.

Gersbach, H. (2005). Democratic mechanisms: Double majority rules and flexible agenda costs. Mimeo, University of Heidelberg.

Green, J. and Laffont, J. (1979). Incentives in Public Decision-Making. North-Holland Publishing Company.

Grossman, G. and Helpman, E. (2001). Special Interest Politics. Cambridge, MA, MIT Press.

Groves, T. (1973). Incentives in teams. Econometrica, 41:617-663.

Guesnerie, R. (1995). A Contribution to the Pure Theory of Taxation. Cambridge University Press.

Hellwig, M. (2003). Public-good provision with many participants. Review of Economic Studies, 70:589-614. 
Heuser, H. (1998). Lehrbuch der Analysis, Teil 1. Teubner; Stuttgart, Leipzig.

Judd, K. (1985). The law of large numbers with a continuum of i.i.d. random variables. Journal of Economic Theory, 35:19-25.

Kalai, E. (2004). Large robust games. Econometrica, 72:1631-1665.

Mailath, G. and Postlewaite, A. (1990). Asymmetric bargaining procedures with many agents. Review of Economic Studies, 57:351-367.

Meirowitz, A. (2005). Polling games and information revelation in the downsian framework. Games and Economic Behavior, 51:464-489.

Mirrlees, J. (1971). An exploration in the theory of optimum income taxation. Review of Economic Studies, 38:175-208.

Piketty, T. (1999). The information-aggregation approach to political institutions. European Economic Review, 43:791-800.

Samuelson, P. (1954). The pure theory of public expenditure. Review of Economics and Statistics, 36:387-389.

Schultz, C. (1996). Polarization and inefficient policies. Review of Economic Studies, 63:331-343.

Weymark, J. (1986). A reduced-form optimal nonlinear income tax problem. Journal of Public Economics, 30:199-217.

Weymark, J. (1987). Comparative static properties of optimal nonlinear income taxes. Econometrica, 55:1165-1185. 